\title{
Energy-Momentum Tensor Improvements in Two Dimensions*
}

\author{
S. Deser \\ Department of Physics, Brandeis University, Waltham, MA 02254-9110 \\ R. Jackiw \\ Center for Theoretical Physics, Laboratory for Nuclear Science and Department of Physics \\ Massachusetts Institute of Technology, Cambridge, MA 02139-4307
}

\begin{abstract}
We discuss some aspects of the two-dimensional scalar field, considering particularly the action for the conformal anomaly as an "improved" gravitational coupling, and the possibility of introducing a dual coupling, which provides a "chiral" energy-momentum tensor improvement.
\end{abstract}

In Memoriam: H. Umezawa

\footnotetext{
*This work is supported in part by funds provided by NSF grant PHY-9315811 and by the U.S. Department of Energy (D.O.E.) under contract \#DE-FC02-94ER40818.
}

Brandeis BRX-TH-384, MIT-CTP-2469

October 1995 
There is very little that is not known about scalar fields and anomalies of conformal (Weyl) symmetry in two spacetime dimensions. In this note, dedicated to the memory of H. Umezawa, who contributed to our understanding of symmetry behavior in field theories, we discuss some less emphasized aspects of the subject, although our remarks may not surprise experts [1]. We shall be especially concerned with the interplay of "energy-momentum tensor improvements" and Schwinger terms / Virasoro algebra central charges, as well as novel, chiral improvements.

I. Consider first the action of a scalar field, coupled non-minimally to gravity

$$
I=-\frac{1}{48 \pi} \int d^{2} x \sqrt{-g}\left(\frac{1}{2} g^{\mu \nu} \partial_{\mu} \varphi \partial_{\nu} \varphi+R \varphi\right)
$$

[Our conventions are: signature $\left.(+,-), R_{\mu \nu}=-\partial_{\alpha} \Gamma_{\mu \nu}^{\alpha}+\ldots, x^{\mu}=(t, \sigma).\right]$ This "improved" scalar field action is of course also the local version of the Polyakov action $I_{P}$.

$$
\begin{gathered}
I_{P}=\frac{1}{96 \pi} \int d^{2} x d^{2} y \sqrt{-g(x)} R(x) K^{-1}(x, y) \sqrt{-g(y)} R(y) \\
-\sqrt{-g(x)} D_{x}^{2} K^{-1}(x, y)=\delta^{2}(x-y)
\end{gathered}
$$

Eq. (1.2a) is obtained from (1.1) upon eliminating $\varphi$ through the usual Gaussian shift, and the field $\varphi$ obeys

$$
D^{2} \varphi=R
$$

Here $D^{2}=g^{\mu \nu} D_{\mu} D_{\nu}$ is the covariant d'Alembertian acting on scalars and the $\delta$-function is a density. The normalization of the integral in (1.1) is chosen to agree with (1.2a). [In fact one may set the $R \varphi$ coefficient to be an arbitrary number $n$, provided the normalizing factor is divided by $n^{2}$; this does not change the overall negative sign in (1.1).]

The metric variation of $I_{P}$ defines the energy-momentum tensor $\theta_{\mu \nu}$,

$$
\begin{gathered}
\frac{2}{\sqrt{-g}} \frac{\delta I_{P}}{\delta g^{\mu \nu}} \equiv \theta_{\mu \nu} \\
\theta_{\mu \nu}=-\frac{1}{48 \pi}\left(\partial_{\mu} \phi \partial_{\nu} \phi-\frac{1}{2} g_{\mu \nu} g^{\alpha \beta} \partial_{\alpha} \phi \partial_{\beta} \phi\right)-\frac{1}{24 \pi}\left(D_{\mu} D_{\nu}-g_{\mu \nu} D^{2}\right) \phi \\
1
\end{gathered}
$$


where $\phi$ is a solution of

$$
D^{2} \phi=R
$$

As is well known, $I_{P}$ emerges when a massless, scalar quantum field $\Phi$, minimally coupled to the metric tensor $g_{\mu \nu}$, is integrated in the functional integral for the partition function [2]. In this framework, $\theta_{\mu \nu}$ may be viewed as the vacuum expectation value of the $\Phi$-field's energy-momentum tensor operator

$$
\Theta_{\mu \nu}=\partial_{\mu} \Phi \partial_{\nu} \Phi-\frac{1}{2} g_{\mu \nu} g^{\alpha \beta} \partial_{\alpha} \Phi \partial_{\beta} \Phi
$$

in the presence of external gravity [3]

$$
\theta_{\mu \nu}={ }_{g}\left\langle\Omega\left|\Theta_{\mu \nu}\right| \Omega\right\rangle_{g}
$$

The evaluation of the partition function leading to (1.2a) or of the matrix element (1.7) requires a choice of regularization. The exhibited formulas reflect the option of maintaining diffeomorphism invariance: under an infinitesmal coordinate transformation,

$$
\delta_{f} x^{\mu}=-f^{\mu}(x)
$$

which is implemented on $g_{\mu \nu}$ by the Lie derivative $L_{f}$ with respect to the vector $f^{\mu}$,

$$
\delta_{f} g_{\mu \nu}=L_{f} g_{\mu \nu}
$$

$I_{P}$ in Eq. (1.2a) is invariant and (1.6) - (1.7) are covariant. However, Weyl invariance is lost: the formal tracelessness of $(1.6)$ is replaced by the anomaly equation, which follows from (1.5)

$$
\theta_{\mu}^{\mu}=\frac{1}{24 \pi} R
$$

This same result may be gotten from the action (1.1), when its fields are subjected to a combined Weyl variation

$$
\begin{aligned}
& \delta g_{\mu \nu}=\chi g_{\mu \nu} \\
& \delta \varphi=\chi \\
& 2
\end{aligned}
$$




$$
\left.2 \frac{\delta I}{\delta \chi}\right|_{\chi=0}=-\frac{1}{24 \pi} \sqrt{-g} R
$$

Therefore, it is recognized that (1.1) is an effective action for the Weyl anomaly - in that sense it is a Wess-Zumino (WZ) - like anomaly action. [Our conventions differ from those used in WZ treatments, where $\delta g_{\mu \nu}=2 \chi g_{\mu \nu}, \delta \varphi=\chi$; this accounts for the relative $\frac{1}{2}$ factor between the two terms in (1.1). Our scaling (1.11) is of course consistent with (1.3).]

An alternative way to see the connection between the nonlocal but purely metric action $I_{P}[g]$ and its local but $\varphi$-dependent WZ formulation (1.1) is the following two-dimensional version of a general construction [5]. Consider the difference

$$
\bar{I} \equiv I_{P}[g]-I_{P}[\tilde{g}], \quad \tilde{g}_{\mu \nu} \equiv g_{\mu \nu} e^{-\varphi} .
$$

Since the combination $\tilde{g}$ is Weyl-invariant by (1.11), it is obvious that $\bar{I}$ yields the same anomaly as $I_{P}[g]$. If one now rewrites $I_{P}[\tilde{g}]$ explicitly using the identity

$$
\sqrt{-\tilde{g}} R(\tilde{g})=\sqrt{-g} R(g)-\sqrt{-g} D^{2} \varphi
$$

and the fact that $K^{-1}$ is insensitive to conformal redefinition of the metric, then (1.13) is precisely (1.1). In principle, one could add any other Weyl-invariant term to (1.13); while this ambiguity is irrelevant here, it is in fact important in higher dimensions [5].

The system (1.1) implies the scalar field equation (1.3), while the metric variation of its action $[c . f .(1.5)]$ is

$$
\begin{gathered}
T_{\mu \nu} \equiv \frac{2}{\sqrt{-g}} \frac{\delta I}{\delta g^{\mu \nu}}=t_{\mu \nu}+\Delta_{\mu \nu} \\
t_{\mu \nu}=-\frac{1}{48 \pi}\left(\partial_{\mu} \varphi \partial_{\nu} \varphi-\frac{1}{2} g_{\mu \nu} g^{\alpha \beta} \partial_{\alpha} \varphi \partial_{\beta} \varphi\right) \quad, \quad \Delta_{\mu \nu}=-\frac{1}{24 \pi}\left(D_{\mu} D_{\nu}-g_{\mu \nu} D^{2}\right) \varphi .
\end{gathered}
$$

The total energy-momentum tensor $T_{\mu \nu}$ consists of the conventional piece $t_{\mu \nu}$, and the improvement $\Delta_{\mu \nu}$ [which survives in the flat space, Minkowski coordinate, limit as $\left.-\frac{1}{24 \pi}\left(\partial_{\mu} \partial_{\nu}-\eta_{\mu \nu} \square\right) \varphi\right]$. Covariant conservation of $T_{\mu \nu}$ is a consequence of the action's coordinate invariance and is guaranteed by (1.3). [In flat space, $\square \varphi$ vanishes and $T_{\mu \nu}$ is also traceless.] 
As is well-known, in this one-component scalar field (rather than string) context, treating the metric as a dynamical variable, thereby setting $T_{\mu \nu}$ to zero, implies that $0=T_{\mu}^{\mu}=$ $\frac{1}{24 \pi} D^{2} \varphi$, and by (1.3), space is flat. Consequently $\varphi$ is a free wave field obeying $\square \varphi=0$. Moreover, for a wave field, $\varphi(x)=\varphi_{+}\left(x^{+}\right)+\varphi_{-}\left(x^{-}\right), x^{ \pm} \equiv \frac{1}{\sqrt{2}}(t \pm \sigma)$ and the remaining $T_{\mu \nu}=0$ equations do not allow any excitations in $\varphi$ : the field profiles are constrained to the forms $\varphi_{ \pm}\left(x^{ \pm}\right)=2 \ln \left(\frac{x^{ \pm}-x_{0}^{ \pm}}{a^{ \pm}}\right)$, where $x_{0}^{ \pm}$and $a^{ \pm}$are constants. We remark that in this model, the coupling of " $\varphi$-matter" to "dynamical gravity" ensures absence of both, contrary to our usual ideas of gravitational coupling and equivalence principle. Note however, the negative sign of the action (1.1), whose consequence is that $\varphi$-matter and gravity possess kinetic terms with opposite signs, and hence their dynamical effects cancel each other.

To avoid triviality, we regard gravity to be a background, and $T_{\mu \nu}$ to be the scalar field's improved stress tensor, even if space is flat (as we shall henceforth assume for simplicity). In that case, $\Delta_{\mu \nu}$ is a superpotential, identically conserved. It plays an essential role in the energy-momentum tensor algebra of the theory, contributing to the Schwinger term or Virasoro central charge.

We recall that in any quantum theory with conventional positivity properties, the equaltime commutator between energy and momentum densities must acquire, in addition to the terms required by Poincaré invariance, a non-vanishing central charge $c$ [6]. In two dimensions, in particular, we must have

$$
\left[T_{00}(\sigma), T_{01}(\tilde{\sigma})\right]=i\left[T_{00}(\sigma)+T_{00}(\tilde{\sigma})\right] \delta^{\prime}(\sigma-\tilde{\sigma})-i c \delta^{\prime \prime \prime}(\sigma-\tilde{\sigma})
$$

where primes denote spatial derivatives and the energy-momentum tensor components are evaluated at a common time $t$, which is suppressed. In addition to the quantal contributions to $c$ from normal-ordering the bilinears $t_{\mu \nu}$ []], there arise "classical" terms coming from $\Delta_{\mu \nu}$. Using the definition (1.16), and the fact that $\frac{\partial}{\partial t} \varphi=\Pi$ is the conjugate momentum variable, we have

$$
\Delta_{00}=-\frac{1}{24 \pi} \varphi^{\prime \prime}, \quad \Delta_{01}=-\frac{1}{24 \pi} \Pi^{\prime}
$$

and so 


$$
i\left[\Delta_{00}(\sigma), \Delta_{01}(\tilde{\sigma})\right]=\frac{1}{(24 \pi)^{2}} \delta^{\prime \prime \prime}(\sigma-\tilde{\sigma})
$$

The remaining commutators in (1.17) are easily seen to reproduce (only) the desired $T_{00}$ terms on the right side. Hence the $W Z$ action (1.1) has indeed, thanks to $\Delta_{\mu \nu}$, given rise to the central charge in (1.17) in a simple free-field context [8].

II. Is $\Delta_{\mu \nu}$ the only possible improvement term for our system? In particular, can one define a chiral counterpart $C_{\mu \nu}=C_{\nu \mu}$ ? Even though there is no spin in two dimensions, there still are right/left movers. Let us first start with free fields in flat space. Just as $\Delta^{\mu \nu}$ can be written as

$$
\Delta^{\mu \nu}=-\frac{1}{24 \pi} \epsilon^{\mu \alpha} \partial_{\alpha} \epsilon^{\nu \beta} \partial_{\beta} \varphi
$$

an odd term involving only two derivatives is uniquely proportional to

$$
C_{\mu \nu} \equiv-\frac{1}{48 \pi}\left(\epsilon_{\mu}^{\alpha} \partial_{\alpha} \partial_{\nu}+\epsilon_{\nu}^{\alpha} \partial_{\alpha} \partial_{\mu}\right) \varphi
$$

(We use $\epsilon^{\alpha \beta}=-\epsilon^{\beta \alpha}, \epsilon^{01}=1$.) Unlike $\Delta_{\mu \nu}, C_{\mu \nu}$ is conserved, not identically, but for wave fields: $\partial_{\mu} C^{\mu \nu}=-\frac{1}{48 \pi} \epsilon^{\nu \alpha} \partial_{\alpha} \square \varphi$. (It is, instead, identically traceless.) This is of course sufficient for our purposes. Let us calculate the relevant components $C_{00}, C_{01}$

$$
C_{00}=-\frac{1}{24 \pi} \Pi^{\prime}=\Delta_{01} \quad, \quad C_{01}=-\frac{1}{24 \pi} \varphi^{\prime \prime}=\Delta_{00}
$$

These equalitites show the duality between the two improvement terms.

Before continuing our development, we must verify that $C_{\mu \nu}$ does not affect the Poincaré generators $\left(P^{\mu}, M\right)$, i.e. that

$$
\delta P^{\mu}=\int d \sigma C^{0 \mu}, \quad \delta M=\int d \sigma\left(\sigma C^{00}-t C^{01}\right)
$$

vanish. This is trivially verified for $\delta P^{\mu}$ and for $\int d \sigma t C^{01}$ by (2.3), provided edge contributions can be dropped. However, there remains

$$
\delta M=\int d \sigma \sigma C^{00}=-\frac{1}{24 \pi} \int d \sigma \sigma \Pi^{\prime} \equiv \frac{1}{24 \pi} \int d \sigma \Pi
$$

which seems not to vanish. To see that it does, we must use the wave equation that ensures conservations of $C_{\mu \nu}$ : any solution of $\square \phi=0$ is the $\operatorname{sum} \varphi_{+}\left(x^{+}\right)+\varphi_{-}\left(x^{-}\right)$, so that 
$\Pi=\varphi_{+}{ }^{\prime}-\varphi_{-}{ }^{\prime}$ and this integrates to zero in the absence of spatial edge terms. (Being a superpotential, the $\Delta_{\mu \nu}$ improvement automatically leaves the Poincaré generators unaffected, absent end-point terms [9].)

One may now consider the general improved energy-momentum tensor,

$$
\bar{T}_{\mu \nu} \equiv t_{\mu \nu}+\alpha \Delta_{\mu \nu}+\beta C_{\mu \nu}
$$

and evaluate (the $\Delta$ - and $C$ - dependent parts of) the commutator (1.17). Using (2.3) and paying attention to the oddness of $\delta^{\prime \prime \prime}$, we find that (1.17) is obeyed in terms of $\bar{T}_{\mu \nu}$, with $c=\frac{1}{(24 \pi)^{2}}\left(\alpha^{2}+\beta^{2}\right)$, results that are indeed exploited when separately calculating left/right contributions to the algebra in more conventional approaches [1].

Given the origin of the superpotential $\Delta_{\mu \nu}$ from a nonminimal covariant coupling to gravity (a general property of true superpotentials), one may ask whether $C_{\mu \nu}$ can likewise be obtained from some original gravitational coupling.

Recall first the $\Delta_{\mu \nu}$ case: we note the variational property

$$
\delta(\sqrt{-g} R)=\frac{-\epsilon^{\mu \alpha} \epsilon^{\nu \beta}}{\sqrt{-g}} D_{\alpha} D_{\beta} \delta g_{\mu \nu}
$$

so that

$$
\delta \int d^{2} x \sqrt{-g} R \varphi=\int d^{2} x \sqrt{-g} \delta g^{\mu \nu}\left(D_{\mu} D_{\nu}-g_{\mu \nu} D^{2}\right) \varphi
$$

as was used for the derivation of $\Delta_{\mu \nu}$ in (1.16). For the chiral version, we need a quantity $C$ to multiply $\varphi$, with variation

$$
\delta \int d^{2} x C \varphi=\int d^{2} x \delta g_{\mu \nu} \epsilon^{\mu \alpha} D^{\nu} D_{\alpha} \varphi
$$

which would give $C_{\mu \nu}$; also the condition $D^{2} \varphi=0$ must be incorporated. In fact a weaker requirement will suffice for our flat space application: the variation (2.7) should hold in the flat space limit.

We can immediately state what this coupling cannot be: since $C_{\mu \nu}$ is not identically conserved, it cannot arise from the variation with respect to $g_{\mu \nu}$ of an invariant action. [Indeed, this is also clear because $\sqrt{-g} R$ is the only geometric scalar density of second derivative order that can be used to multiply $\varphi$ [10].] 
An imperfect solution to these requirements is constructed in terms of the quantity $R^{\mu}$, whose divergence is the Euler density.

$$
\partial_{\mu} R^{\mu}=\sqrt{-g} R
$$

Of course, $R^{\mu}$ is ambiguous up to terms of the form $\epsilon^{\mu \nu} \partial_{\nu} r$, which would not contribute to (2.8). $R^{\mu}$ cannot be presented explicitly and locally in terms of the generic metric $g_{\mu \nu}$ and its derivatives $\partial_{\alpha} g_{\mu \nu}$ [11]; rather it is necessary to parametrize $g_{\mu \nu}$. Defining the unimodular metric

$$
\gamma_{\mu \nu}=g_{\mu \nu} / \sqrt{-g}, \quad \sqrt{-g} \equiv e^{\sigma}
$$

and parametrizing $\gamma_{\mu \nu}$ and its inverse $\gamma^{\mu \nu}$, by writing their light cone components as

$$
\begin{aligned}
& \gamma_{++}=-\gamma^{--}=e^{\alpha} \sinh \beta \\
& \gamma_{--}=-\gamma^{++}=e^{-\alpha} \sinh \beta \\
& \gamma_{+-}=\gamma_{-+}=\gamma^{+-}=\gamma^{-+}=\cosh \beta
\end{aligned}
$$

gives for $R^{\mu}$

$$
R^{\mu}=\gamma^{\mu \nu} \partial_{\nu} \sigma+\partial_{\nu} \gamma^{\mu \nu}-\epsilon^{\mu \nu}(\cosh \beta-1) \partial_{\nu} \alpha
$$

The explicit parametrization (2.10) is needed to present the last term in (2.11) [12].

Even though the last contribution in (2.11) to $R^{\mu}$ is not expressible in terms of $g_{\mu \nu}$ or $\gamma_{\mu \nu}$, its arbitrary variation satisfies a formula involving only $\gamma_{\mu \nu}$

$$
\delta\left[\epsilon^{\mu \nu}(\cosh \beta-1) \partial_{\nu} \alpha\right]-\partial_{\nu}\left[\epsilon^{\mu \nu}(\cosh \beta-1) \delta \alpha\right]=-\frac{1}{2} \gamma^{\mu \nu}\left(\partial_{\alpha} \gamma_{\nu \beta}+\partial_{\beta} \gamma_{\nu \alpha}-\partial_{\nu} \gamma_{\alpha \beta}\right) \delta \gamma^{\alpha \beta}
$$

The second term on the left is a curl and so will not contribute; the right side may be written as $-\gamma_{\alpha \beta}^{\mu} \delta \gamma^{\alpha \beta}$, where $\gamma_{\alpha \beta}^{\mu}$ is the Christoffel affinity of the $\gamma_{\mu \nu}$ metric: $\gamma_{\alpha \beta}^{\mu}=\left.\Gamma_{\alpha \beta}^{\mu}\right|_{g_{\mu \nu}=\gamma_{\mu \nu}}$.

We remark that the last term in (2.11) naturally defines a 1 -form $a \equiv(\cosh \beta-1) d \alpha$ and the 2-form $\omega=d a=\sinh \beta d \beta d \alpha$. These are recognized as the canonical 1-form and the symplectic 2 -form, respectively, for $\operatorname{SL}(2, R)$. Indeed $\omega$ also equals $\frac{1}{2} \epsilon_{a b c} \xi^{a} d \xi^{b} d \xi^{c}$, where $\xi^{a}$ is 
a three-vector on a hyperboloid $=\mathrm{SL}(2, R) / \mathrm{U}(1):\left(\xi^{1}\right)^{2}-\left(\xi^{2}\right)^{2}-\left(\xi^{3}\right)^{2}=-1$. Effectively, $\omega$ is the Kirillov-Kostant 2-form on $\operatorname{SL}(2, R)$ 13.

While the divergence of $R^{\mu}$ is the scalar curvature density, $R^{\mu}$ itself is not a vector density under coordinate transformations. Rather for the infinitesimal diffeomorphism (1.8) one verifies that there is an additional, identically conserved, non-tensor term in the transformation law

$$
\begin{gathered}
\delta_{f}\left(R^{\mu} / \sqrt{-g}\right)=L_{f}\left(R^{\mu} / \sqrt{-g}\right)+\epsilon^{\mu \nu} \partial_{\nu} \Delta_{f} / \sqrt{-g} \\
\Delta_{f} \equiv\left(\partial_{+}-e^{\alpha} \tanh \frac{\beta}{2} \partial_{-}\right) f^{+}-\left(\partial_{-}-e^{-\alpha} \tanh \frac{\beta}{2} \partial_{+}\right) f^{-} .
\end{gathered}
$$

Consequently, a world scalar action equivalent to 2.6b may be constructed by coupling vectorially $R^{\mu}$ to a scalar field $\varphi, I_{V}=\int d^{2} x R^{\mu} \partial_{\mu} \varphi$. [The formula (2.6b) is preferred in that no derivatives act on $\varphi$ and the symplectic structure is preserved.] But now we see that the axial action

$$
I_{C}=\int d^{2} x \frac{\epsilon^{\mu \nu}}{\sqrt{-g}} R_{\mu} \partial_{\nu} \varphi=\int d^{2} x \varphi \epsilon^{\mu \nu} \partial_{\mu}\left(\frac{R_{\nu}}{\sqrt{-g}}\right)
$$

will be insensitive both to the variation (2.13), and to the ambiguity $\epsilon^{\mu \nu} \partial_{\nu} r$ in $R^{\mu}$ if $\varphi$ satisfies $D^{2} \varphi=0$ [The last form of (2.14) preserves the symplectic structure of the $\varphi$ field.] Thus we attempt generating $C_{\mu \nu}$ from $I_{C}$.

When $I_{C}$ is varied, one finds using (2.12) that

$\delta I_{C}=\int d^{2} x \delta g_{\mu \nu}\left[\epsilon^{\mu \alpha}\left(D^{\nu}+\frac{R^{\nu}}{\sqrt{-g}}\right) D_{\alpha} \varphi-g^{\mu \nu} \frac{R_{\alpha}}{\sqrt{-g}} \epsilon^{\alpha \beta} \partial_{\beta} \varphi\right]-\int d^{2} x \sqrt{-g} \delta \alpha[\cosh \beta-1] D^{2} \varphi$

The second integral may be dropped when $D^{2} \varphi=0$, and the desired result (2.7) is achieved, in flat space where $R^{\mu}$ vanishes. Thus the chiral improvement (2.2) may be derived by varying $\frac{1}{48 \pi} I_{C}$, and passing to flat space. But what remains unsatisfactory about the $C_{\mu \nu}$ construction, as compared to that of $\Delta_{\mu \nu}$, is our inability to present a single action of the form $I[\varphi]+I_{C}$, such that its $\varphi$-variation implies the required $D^{2} \varphi=0$ and flatness conditions.

The explicit tracelessness of $C_{\mu \nu}$ is now also understood: from (2.11) and (2.14) we see that $I_{C}$ does not depend on the conformal factor $\sigma$, and is therefore Weyl invariant, even when it is not diffeomorphism invariant for $D^{2} \varphi \neq 0$. 
We conclude that while the two improvements we have discussed are both permitted (and useful) additions to the flat space scalar field's stress tensor, they have quite different status when related to underlying gravitational couplings. 


\section{REFERENCES}

[1] For a review, see for example D. Friedan, E. Martinec and S. Shenker, Nucl. Phys. B271, $93(1986)$.

[2] A. Polyakov, Mod. Phys. Lett. A2, 893 (1987); Gauge Fields and Strings (Harwood, New York, NY, 1987); see also M. Duff, Nucl. Phys. B125, 334 (1977).

[3] This alternative determination of $\theta_{\mu \nu}$ was given by M. Bos, Phys. Rev. D 34, 3750 (1986).

[4] It is interesting to note that alternative procedures for handling infinities are available and that it is possible to maintain Weyl invariance, at the expense of diffeomorphism invariance. Nevertheless, diffeomorphisms with unit Jacobian, i.e., in (1.8) $\partial_{\mu} f^{\mu}=0$, are maintained. With this approach, the energy-momentum tensor remains traceless,

but its divergence becomes $-\frac{1}{48 \pi} \partial_{\mu} R$, a formula whose structure is in fact dictated by the restricted diffeomorphism symmetry. "Physical" results, like two-dimensional Hawking radiation, remain unaffected. For details see: D. Karakhanyan, R. Manvelyan and R. Mkrtchyan, Phys. Lett. B329, 185 (1994); G. Amelino-Camelia, D. Bak and D. Seminara, Phys. Lett. B354, 213 (1995); J. Navarro-Salas, M. Navarro and C. Talavera, Phys. Lett. B356, 217 (1995); and G. Amelino-Camelia and D. Seminara, MIT preprint MIT-CTP-2443.

[5] S. Deser and A. Schwimmer, in preparation.

[6] D. Boulware and S. Deser, J. Math. Phys. 8, 1468 (1967).

[7] In the two-dimensional case, these are finite and were calculated long ago by S. Fubini, A. Hanson and R. Jackiw, Phys. Rev. D 7, 1732 (1972) as well as by S. Ferrara, R. Gatto and A. Grillo, Nuevo Cim. 12A, 959 (1972).

[8] That the improved energy-momentum tensor possess a canonical triple-derivative Schwinger term in its equal time commutator algebra was noted by C. Callan, S. Coleman and R. Jackiw, Ann. Phys. (NY) 59, 42 (1970). 
[9] The improvement does modify the dilation and special conformal generators in dimensions higher than two, and for the Liouville theory in two dimensions. In these instances, the unimproved energy-momentum tensor is not traceless, so that dilation and special conformal generators cannot be given by moments of this energy-momentum tensor, see e.g. Ref. [8] and Jackiw in Progress in Quantum Field Theory, H. Ezawa and S. Kamefuchi, eds. (North-Holland, Amsterdam, Netherlands 1986).

[10] R. Jackiw, C. Teitelboim in Quantum Theory of Gravity, S. Christensen, ed. (Adam Hilger, Bristol, UK, 1984).

[11] In the Zweibein $\left(e_{\alpha}^{a}\right)$-connection $\left(\omega_{\mu} \equiv \omega_{\mu a b} \epsilon^{a b}\right)$ form of the Euler density $\sqrt{-g} R=$ $\epsilon^{\mu \nu} \partial_{\mu} \omega_{\nu}, R^{\mu}$ is explicitly defined (up to the ambiguity $\epsilon^{\mu \nu} \partial_{\nu} r$ ) without parametrization. However, $R^{\mu}$ now no longer depends solely on the metric, but involves the Zweibein in an essential way, since the connection is $\omega_{\nu}=\frac{\epsilon^{\alpha \beta}}{\sqrt{-g}} \partial_{\beta} g_{\alpha \nu}+\epsilon_{a b} g^{\alpha \beta} e_{\alpha}^{a} \partial_{\nu} e_{\beta}^{b}$. The first term is metric, but the second one clearly depends on the essentially non-metric part of the Zweibein.

[12] This is analogous to what happens with a Chern-Simons term. Upon performing a gauge transformation with a gauge function $U$, the Chern-Simons term changes by a total derivative. However, direct evaluation of the gauge response includes the expression $\omega=\frac{1}{24 \pi^{2}} \operatorname{tr} \epsilon^{\alpha \beta \gamma} U^{-1} \partial_{\alpha} U U^{-1} \partial_{\beta} U^{-1} \partial_{\gamma} U$, which can be recognized as a total derivative only after $U$ is explicitly parametrized. For example, in $\mathrm{SU}(2), U=\exp \theta, \theta=\theta^{a} \sigma^{a} / 2 i$, and $\omega=\partial_{\alpha} \omega^{\alpha}$ where $\omega^{\alpha}=\frac{1}{4 \pi^{2}} \operatorname{tr} \epsilon^{\alpha \beta \gamma} \theta \partial_{\beta} \theta \partial_{\gamma} \theta\left(\frac{|\theta|-\sin |\theta|}{|\theta|^{3}}\right)$ with $|\theta| \equiv \sqrt{\theta^{a} \theta^{a}}$; see Jackiw in S. Treiman, R. Jackiw, B. Zumino and E. Witten, Current Algebra and Anomalies, (Princeton University Press / World Scientific, Princeton, NJ / Singapore, 1985).

[13] We thank V. P. Nair for pointing this out. 